\title{
Observation of positron trapping and air condensation in porous silicon by angular correlation annihilation radiation
}

\author{
C.C. Haung, I.M. Chang, Y.F. Chen*, P.K. Tseng \\ Department of Physics, National Taiwan University, Taipei, Taiwan
}

Received 2 April 1996; revised 17 June 1996

\begin{abstract}
The momentum spectrum of porous silicon has been measured by two dimensional angular correlation of positron-electron annihilation radiation (2D-ACAR). Comparing with the bulk silicon two extra peaks with different linewidths have been found. A very interesting phenomenon is observed that when the sample is placed at $15 \mathrm{~K}$ in a vacuum of $10^{-6}$ Torr, the intensity of the narrower peak decreases and its linewidth increases, while that of the wider peak remains unchanged. When the temperature is restored to $300 \mathrm{~K}$, the spectrum recovers its original feature. From this observation, we attribute the narrower peak to the positronium trapped in the etched pores and not on the surface of pores. The wider peak is suggested to be due to the positron trapped in the silicon nanocrystal. With a simple model which assumes an infinite spherical potential well, we estimate the average size of the etched pores to be about $24 \AA$.
\end{abstract}

Keywords: Porous silicon; Positron; Microstructure

Since the discovery of strong luminescence [1], the porous silicon formed by electrochemical dissolution in HF acid has been widely studied due to the possibility of its application in optoelectronic devices. However, the origin of the luminescence is not clear at present. In order to understand the mechanism of luminescence, it is important to study the microstructure of porous silicon. The direct methods for the observation of the microstructure include high resolution transmission electron microscope, scanning electron microscope [2], and scanning tunneling microscope [3]. Even though these measurements can well resolve the morphologies of the studied materials, they are insufficient to determine the microstructure of porous silicon and find the mechanism of the photoluminescence because of the nanometer size (or

\footnotetext{
* Corresponding author.
}

even smaller) and the complexity of the tree-like porous structure.

The positron, because of having positive charge, is repelled from the ion cores and shows a very good sensitivity to defects or voids in materials [4]. Thus, the techniques related to the positron annihilation are very powerful tools for the study of porous materials and reveal useful information. In the positron annihilation lifetime measurement, Cruz et al. found that the intermediate positron lifetime strongly depends on the sample preparing conditions and annealing temperatures [5]. Itoh et al. found a long-lived ( $25 \mathrm{~ns}$ ) and sharp Doppler broadening distribution which shows the existence of positronium [6]. Suzuki et al. observed a dramatic change in lifetime with sample temperature and annealing temperature [7]. However, the complex porous structure creates a multicomponent positron state, and obscures a clear physical explanation. The positron may annihilate in the 
nanocrystal of porous silicon directly, get trapped in the vacancy, or capture an electron forming positronium trapped inside or on the surface of the pores. To use positron for the study of the microstructure, the most important thing is to identify the annihilating site. The corresponding average size of the free volume of the annihilating site can be estimated from the positron spectrum. However, the previous positron studies on porous silicon were unable to make a clear identification. In this paper, the positron annihilation spectrum was measured by $2 \mathrm{D}$-ACAR which is known to have higher resolution than that of the Doppler broadening experiment used previously for the study of porous silicon. We clearly observed two components in the obtained spectra due to the existence of porous silicon. We also present a method to identify the positron annihilation sites by observing the slow change of electron-positron momentum distribution at low temperature in a vacuum chamber. The reason for performing measurement in vacuum is to prevent air condensation which may offer enough liquid to generate a positronium bubble state [8] and make positive identification difficult. We found that when the sample is placed at $15 \mathrm{~K}$ in a vacuum of $10^{-6}$ Torr, the gas molecules of air condense on the surface of porous silicon slowly. The air condensation will change the component of the spectrum contributed by positron annihilating in the pores or on the porous surface. From our measurement, the positronium annihilation in the etched pores is identified, and the corresponding average size is estimated. In addition, the possibility of a positron getting trapped in the silicon nanocrystal is also suggested.

The wafers used were $(100)$ oriented p-type silicon single crystals with resistivities of $10-20 \Omega \mathrm{cm}$. The silicon crystals were evaporated with aluminum as electrode. The porous silicon is formed by anodizing in hydrofluoric acid $\left(\mathrm{HF}: \mathrm{H}_{2} \mathrm{O}\right.$ : $\mathrm{C}_{2} \mathrm{H}_{5} \mathrm{OH}=1: 1: 2$ ) under a constant current $25.5 \mathrm{~mA} / \mathrm{cm}^{2}$. The etching depth is about $2000 \AA$.

The momentum distribution of porous silicon is measured by $2 \mathrm{D}-\mathrm{ACAR}$ at room temperature or $15 \mathrm{~K}$ with a vacuum of $10^{-6}$ Torr. The system uses two sets of $\mathrm{NaI}(\mathrm{Tl})$ crystal scintillator $(28.8 \mathrm{~cm}$ radius and $1 \mathrm{~cm}$ thickness) combined with 61 pieces of R1410(Hamamatsu) photomultiplier tubes. The angular resolution is about $0.6 \times 0.6 \mathrm{mrad}$. The positron comes from a $30 \mathrm{mCi} \mathrm{Na}^{22}$ source and is focused by one tesla magnetic field. The 2D-ACAR spectrum,

$N\left(p_{x}, p_{y}\right)=\int \rho(p) \mathrm{d} p_{z}$,

is the projection of the electron-positron momentum density $\rho$ onto $p_{x}-p_{y}$ momentum plane. The typical spectrum was collected by about $5 \times 10^{6}$ to $10^{7}$ events depending on the measuring time. All the spectra have been repeated and they show a good reproducibility.

Fig. 1 shows the electron-positron momentum distribution of porous silicon at room temperature. This spectrum consists of the positron annihilating in porous silicon and silicon wafer. It has been circularly averaged to reduce the statistical error because the central peaks which come from the contribution of positron annihilation in porous layer do not exhibit clear difference between the $p x$ and $p y$ directions. The result can be compared with the spectrum of pure silicon wafer which was measured under the same condition as shown in Fig. 1. After subtracting the contribution of the silicon wafer, we can see that the porous silicon spectrum clearly consists of two peaks with different line widths as shown in Fig. 2. It is interesting that we can observe the positron annihilation in the porous layer with a thickness of only $2000 \AA$. Positrons

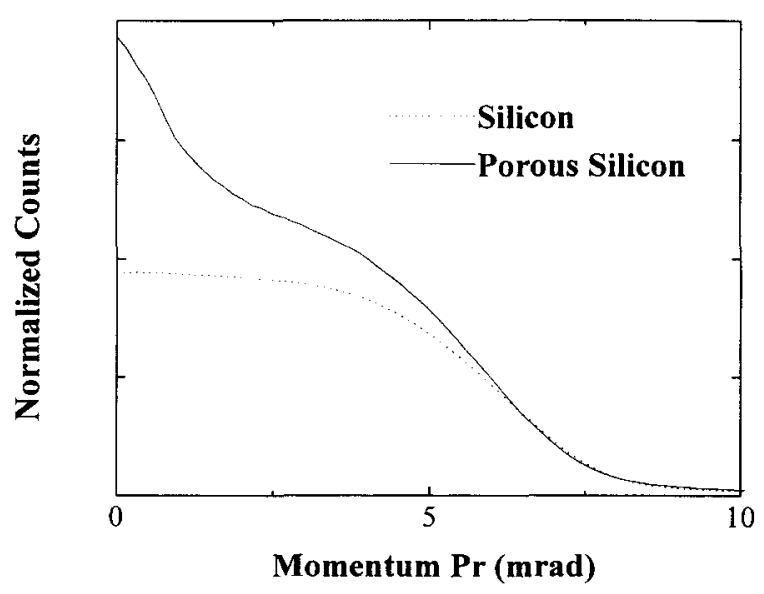

Figure 1. 


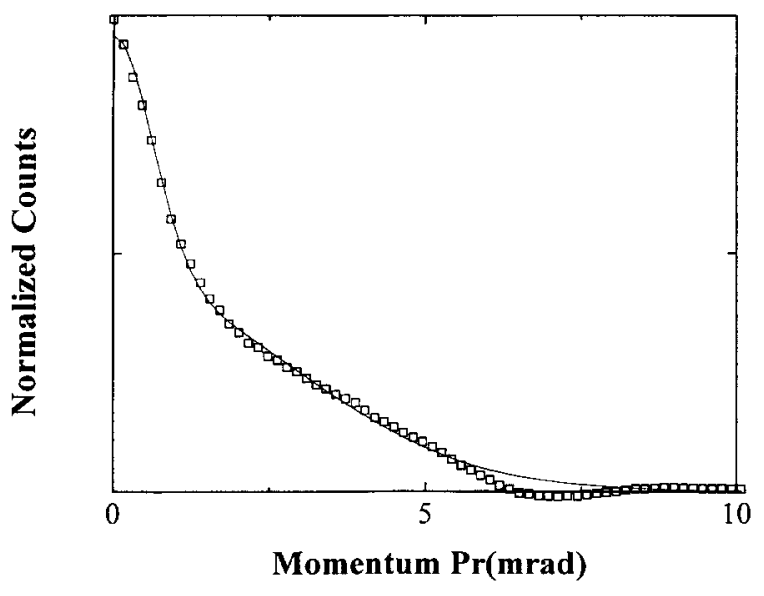

Figure 2.

have a large penetration depth of $100 \mu \mathrm{m}$ and hence one should expect that most of the positrons have penetrated the layer of porous $\mathrm{Si}$. This fact can be attributed to the existence of back diffusion of thermalized positrons [9] and a large amount of free regions and defects in porous layer to trap the positrons escaping from the silicon bulk. The most interesting observation of the spectrum is that when the measurement is performed at $15 \mathrm{~K}$ in a vacuum of $10^{-6}$ Torr, the intensity of the narrower peak decays with a half-life $t_{1 / 2}=12 \mathrm{~h}$, while the wider peak remains unchanged. If the measurement is performed at room temperature again, the narrower peak recovers to its original value. This reversible process can be repeated many times.

The above observation suggests that the obtained spectrum can be decomposed into two gaussian peaks. We can fit the spectrum by

$$
N\left(p_{\mathrm{r}}\right)=a_{\mathrm{n}} \exp \left(-\frac{p_{\mathrm{r}}^{2}}{2 \sigma_{\mathrm{n}}^{2}}\right)+a_{\mathrm{w}} \exp \left(-\frac{p_{\mathrm{r}}^{2}}{2 \sigma_{\mathrm{w}}^{2}}\right),
$$

where $p_{\mathrm{r}}$ is the momentum, $a_{\mathrm{n}}$ and $a_{\mathrm{w}}$ are weighting parameters, $\sigma_{\mathrm{n}}$ and $\sigma_{\mathrm{w}}$ are the standard deviations of the narrower peak and the wider peak, respectively. The spectrum was well fitted by Eq. (2) as shown in Fig. 2. Fig. 3 shows the intensity and full width at half maximum (FWHM) of the narrower peak varying with time and Fig. 4 shows the result of the wider peak. The first data point is measured before cooling and the last data point is measured after the temperature is restored to the room tem-

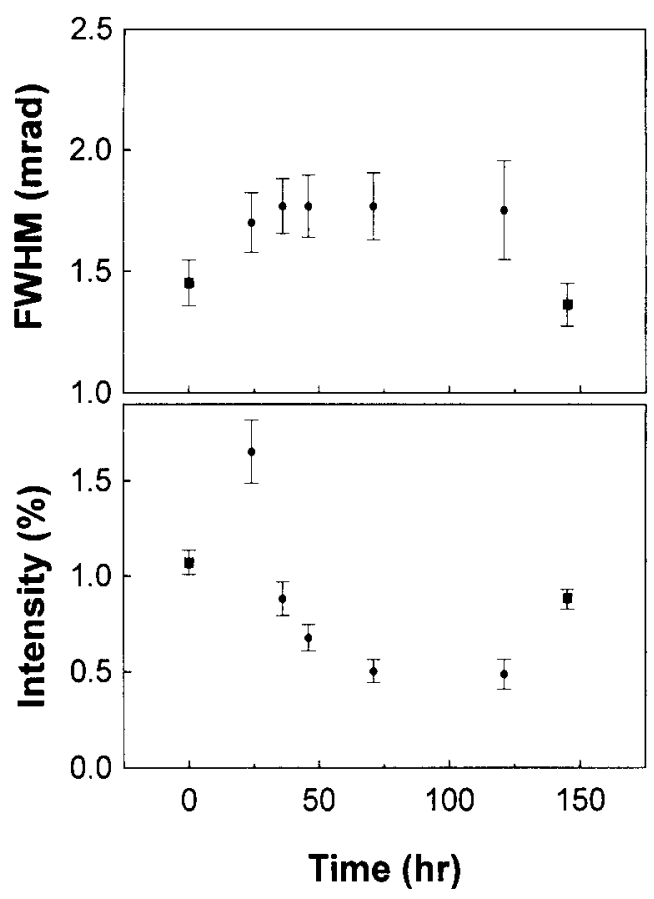

Figure 3.

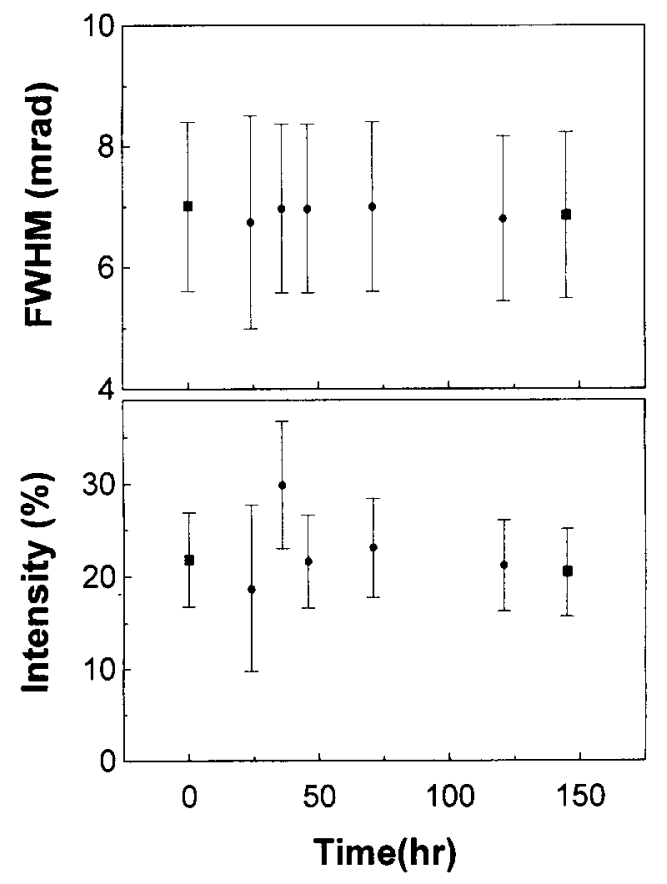

Figure 4. 
perature. From Fig. 3 we can see clearly that when the sample was placed at $15 \mathrm{~K}$, the FWHM of the narrower peak became larger and the intensity $I_{\mathrm{n}}$ increased first and then began to decay. After the sample returned to the room temperature, the intensity and FWHM of the narrower peak recovered to the original value. In the same process, the wider peak does not change with time in all the measurements.

The narrower peak in our measured 2D-ACAR spectrum is the result of positronium formation in porous silicon which can be correlated to the longlived component in the lifetime spectrum obtained by Suzuki et al. [7]. In their report, the positronium is assumed to be formed at the surface of etched pores. Here, we provide further evidences to show that the positronium is trapped inside the pores and not on the surface of the pores. The first evidence comes from the increasing of the FWHM of the narrower peak with the time of keeping the sample in the vacuum of $10^{-6}$ Torr as shown in Fig. 3. We explain this behavior in terms of the condensation of the air molecules in the vacuum chamber. Air condensation shields the pores, and hence reduces the size of the free volume. The decreasing of the available free volume for the trapped positronium will increase the FWHM of the corresponding spectrum [4], and it makes the narrower component become wider. Thus, the experimental fact can be explained. If the positronium were trapped on the surface, the momentum spectrum, which depends on the perpendicular and parallel linewidths to the surface [10], will become sharper at lower temperature because of the decrease of the parallel linewidth caused by lower thermal velocity. This expectation is contrary to our result. Furthermore, when the sample was placed at $15 \mathrm{~K}$, in the beginning a little air condenses in the pores and forms a monolayer liquid. This two dimensional liquid will help the positronium formation by playing the role of a recoil agent to absorb the surplus momentum of positronium at first [11]. Then the air continues to condense and begins to fill the smaller pores and restricts the formation of positronium. Thus, the intensity will increase in the beginning and then decrease. This behavior is again consistent with our result. However, if the positronium were trapped on the surface of the pores, the large surface area of porous silicon should help the formation of positronium after air condensation and we would have found a dramatic increase in the intensity, which is opposite to what we generally observe. Therefore, we can conclude that the positronium is trapped inside the free volume of the pores.

If we assign the peak as due to the positronium trapping in the smaller pores and simplify the potential seen by the positronium as a spherical hole with an infinite potential outside. We can estimate the void size by [9]

$R(\AA)=\frac{16.6}{\theta_{1 / 2}(\mathrm{mrad})}$,

where $R$ is the radius of hole and $\theta_{1 / 2}$ is the FWHM of the peak. This relation has been applied successfully to estimate the bubble sizes in many different materials, such as organic liquid, Zeolite, molecular solid, amorphous silicon and glasses [12-14]. It is found that the obtained value has a $15 \%$ variation compared to other techniques. Thus, it should be also appropriate to apply to the study of porous silicon. By Eq. (3), the estimated average size $2 R$ of the pores is about $24 \pm 4.0 \AA$ at room temperature and $18.6 \pm 2.9 \AA$ at $15 \mathrm{~K}$. This difference can be attributed to the air condensation in the pores where positronium is trapped. The obtained size here is only the average value of the pores because one should expect that there has to be a distribution of the void size in porous silicon. This value is close to the result of $20 \AA$ estimated by Suzuki et al., where they used the pick-off positronium lifetime component and the free volume trapped model [8].

The identification of the wider peak is much more difficult. It may correlate with the second component in the lifetime spectrum observed by Suzuki et al. [7]. It has been attributed to the trapping of positrons by vacancy-type defects. However, because the wider component does not vary with temperature, this result is not consistent with positron trapping in the defects of silicon [15]. Thus, the positrons trapped at vacancy defects cannot be the origin of the wider spectrum. A possible origin is the positrons trapped in the silicon nanocrystal. In the nanocrystal region there is no 
free volume for air condensation, and hence the trapping probability does not change in the measured temperature region [13]; thus the behavior of the trapped positrons remains unchanged, which is just what we have observed here. It is valuable if we can obtain the size of the nanocrystal from the spectrum and compare with the data by other techniques. However, the details of the size of the nanocrystal in the 2D-ACAR measurement still needs further theoretical study.

In conclusion, we have studied the microstructure of porous silicon by 2D-ACAR measurement. Two central peaks were found in the momentum spectrum. When the sample is placed at low temperature, an interesting phenomenon is found: due to the effect of air condensation, the spectrum of the narrower peak changes with time, while the wider peak remains unchanged. This observation helps us in our assignment of the positronium and positron annihilating sites. In addition, from the high resolution of the 2D-ACAR spectrum, the estimated average size of the etched pores is about $24 \AA$. Although we still do not know the exact state of the wider peak, the most possible origin, however, can be attributed to positrons trapped in the silicon nanocrystal. Corresponding theoretical calculations will further enhance our understanding of the size confinement by the technique of 2D-ACAR. Thus, our measurement not only reveals the microstructure of porous silicon, but it also suggests that the technique of 2D-ACAR is a useful tool for the study of nanocrystals.
This work is partially supported by the National Science Council of the Republic of China.

\section{References}

[1] L.T. Canham, Appl. Phys. Lett. 57 (1990) 1046.

[2] R.L. Smith and S.D. Collins, J. Appl. Phys. 71 (1992) R1.

[3] V.P. Parkhutik and J.M. Albella, J.M. Martinez-Duart, J.M. Gomez-Rodriguez, A.M. Baro and V.I. Shershulsky, Appl. Phys. Lett. 62 (1993) 366.

[4] S.J. Wang and Y.C. Jean, in: Positron and Positronium Chemistry, eds. D.M. Schrader and Y.C. Jean (Elsevier, Amsterdam, 1988) p. 255.

[5] R.M. De la Cruz and R. Pareja, Phys. Stat. Sol. A 111 (1989) 463.

[6] Y. Itoh, H. Murakami and A. Kinoshita, Appl. Phys. Lett. 63 (1993) 2798.

[7] R. Suzuki, T. Mikado, H. Ohgaki, M. Chiwaki and T. Yamazaki, Phys. Rev. B 49 (1994) 17484.

[8] H. Nakanishi and Y.C. Jean, in: Positron and Positronium Chemistry, eds. D.M. Schrader and Y.C. Jean (Elsevier, Amsterdam, 1988) p. 159.

[9] P.J. Schuitz and K.G. Lynn, Rev. Mod. Phys. 60(3) (1988) 701.

[10] K.O. Jensen, M. Eldrup, B.N. Singh, S. Linderoth and M.D. Bentzon, J. Phys. F 18 (1988) 1091.

[11] P. Rice-Evans and K.U. Rao, Phys. Rev. Lett. 61 (1988) 581.

[12] C.V. Briscoe, S.I. Choi and A.T. Stewart, Phys. Rev. Lett. 20 (1968) 493.

[13] Y.J. He, M. Hasegawa, R. Lee, S. Bergo, D. Adler and A.L. Jung, Phys. Rev. B 33 (1986) 5924.

[14] A. Uedono and S. Tanigawa, Jpn. J. Appl. Phys. 32(1) (1993) 2687.

[15] M. Shimotomai, Y. Ohgino, T. Mihara, K. Inoue and M. Doyama, in: Positron Annihilation, eds. P.G. Coleman, S.C. Sharma and L.M. Diane (North-Holland, Amsterdam, 1983), p. 679 . 